\title{
A comparision of Hodgkin-Huxley and soliton neural theories
}

\author{
R. Appali, S. Petersen, and U. van Rienen \\ Institute of General Electrical Engineering, Chair of Electromagnetic Field Theory, University of Rostock, \\ Justus-von-Liebig-Weg 2, 18059 Rostock, Germany
}

\begin{abstract}
Hodgkin and Huxley were the pioneers to abstract biological neuron as an electric circuit and nerve signal as the voltage impulse. The Hodgkin-Huxley theory (Hodgkin and Huxley, 1952) has set the direction and defined the goals for much of the ensuing research in biophysics. However, in 2005, T. Heimburg and A. D. Jackson, biophysicists from Copenhagen proposed a new neural theory called Soliton theory (Heimburg and Jackson, 2005). In this theory, the nerve conduction is proposed as a density wave.

In this paper, Hodgkin-Huxley and Soliton theories are described and a theoretical comparison has been carried out throughout the analysis of the theories and models.
\end{abstract}

\section{Introduction}

Nerves are the information transmitter lines from the brain to organs. The nerves are described as the bundle of discrete individual cells called neurons. These individual cells transmit the information as signals.

The main parts of a neuron are the cell body (soma), signal receiving ends (dendrites), signal transmitter (axon), and connecting ends to other neurons or glands (synapses). The scientific model of the neuron was first given in electrical terms by Hodgkin and Huxley (Hodgkin and Huxley, 1952). Hodgkin and Huxley conducted the experiments on nonmyelinated giant axon of squid to study the neuronal properties. The Hodgkin-Huxley equations (Hodgkin and Huxley, 1952) are the starting point for detailed neuronal models.

\section{The Hodgkin-Huxley theory}

The theory was the summation of active experimental and theoretical association of Hodgkin and Huxley. Their five publications in 1952 in the Journal of Physiology are quite

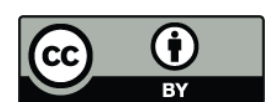

Correspondence to: R. Appali

(revathi.appali@uni-rostock.de) renowned. The four key research developments which led to the theory were: (Häusser, 2000)

- Firstly, Cole and Curtis demonstrated that the action potential is associated with a large increase in membrane conductance (Cole and Curtis, 1939).

- Secondly, Hodgkin and Huxley made the first intracellular recording of an action potential (Hodgkin and Huxley, 1952). This demonstrated that the action potential exceeds zero $\mathrm{mV}$, denying Bernstein's hypothesis (Bernstein, 1902) that the underlying increase in membrane permeability is non-selective.

- Hodgkin and Katz (Hodgkin and Katz, 1949) explained the overshooting action potential as the result of an increase in sodium permeability approving the work of Overton (Overton, 1902).

- Finally, Hodgkin, Huxley and Katz developed the voltage-clamp circuit to facilitate quantitative measurement of ionic currents from squid axon.

The step depolarization of squid axon triggering an inward current followed by an outward current was then proved by Hodgkin and Huxley. With the aid of ionic substitution, they demonstrated that this net current could be separated into two distinct components, a fast inward current carried by $\mathrm{Na}^{+}$ions, and a more slowly activating outward current carried by $\mathrm{K}^{+}$ions. Using ingenious voltage-clamp protocols, they concluded that these two currents result from independent permeability mechanisms for $\mathrm{Na}^{+}$and $\mathrm{K}^{+}$with conductance changing as a function of time and membrane potential. This striking conceptual breakthrough was then termed as the "ionic hypothesis" (Häusser, 2000).

The three different types of ion current, viz., sodium, potassium, and a leak current that consists mainly of $\mathrm{Cl}^{-}$ ions contribute to the voltage signal in the neuron. The flow of these ions through the cell membrane is controlled by their respective voltage-dependent ion channels. The leak current takes care of other channel types which are not described in particular. The most remarkable achievement of this theory 


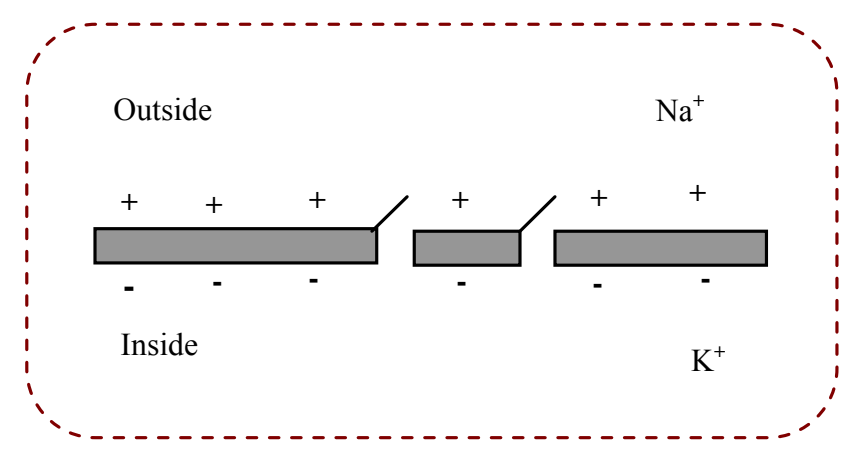

Fig. 1. Schematic model of Hodgkin-Huxley theory (modified from Gerstner et al., 2002).

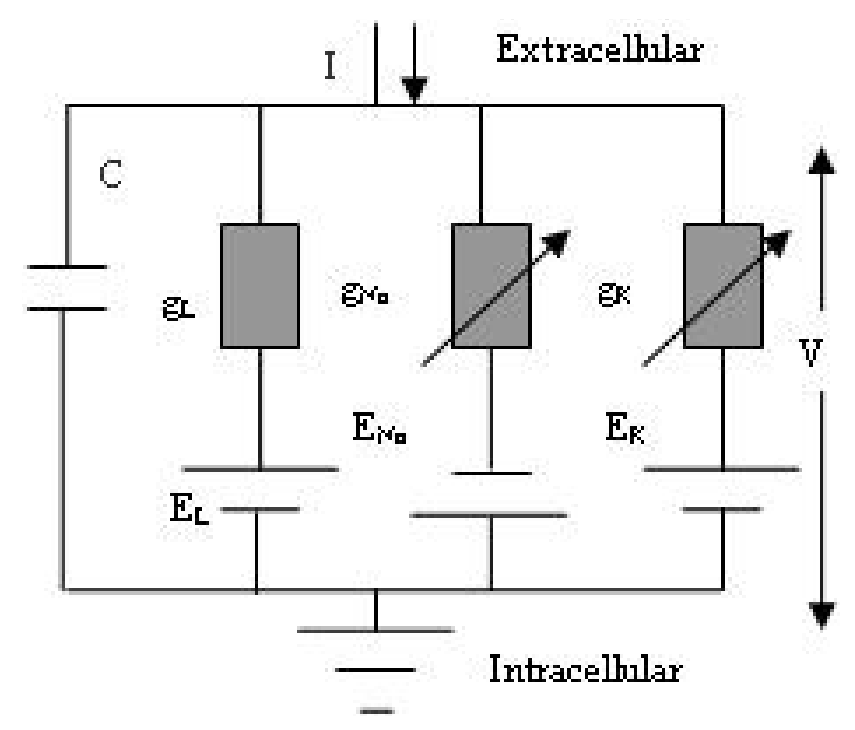

Fig. 2. Equivalent circuit of H-H model (Hodgkin and Huxley, 1952).

was the empirical representation of the experimental data in a quantitative model (Hodgkin and Huxley, 1952), the very first complete description of the excitability of single neuron.

\subsection{The model}

The Hodgkin-Huxley model (H-H model) can be explained with the help of Fig. 1. The semi-permeable cell membrane separates the interior of the cell from the extracellular liquid and acts as a capacitor. If an input current $I(t)$ is injected into the cell, it may add further charge on the capacitor $\mathrm{C}$, or leak through the channels in the cell membrane. Because of active ion transport through the cell membrane, the ion concentration inside the cell is different from that of the ion concentration in extracellular liquid. The Nernst potential generated by the difference in ion concentrations is represented by a battery. The equivalent circuit is shown in Fig. 2.

As mentioned above, the Hodgkin-Huxley model describes three types of channels. All channels may be char-

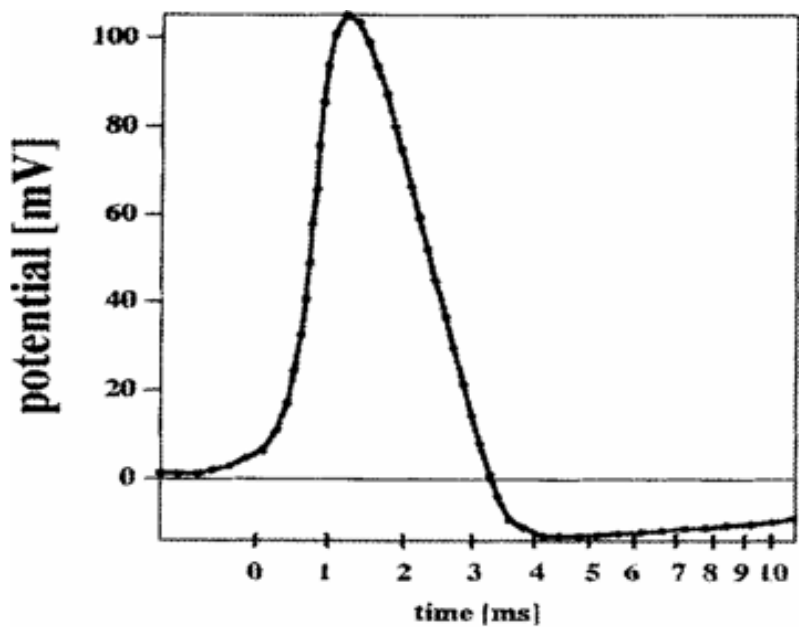

Fig. 3. Action potential adapted from the original paper of Hodgkin and Huxley.

acterized by their resistance or, equivalently, by their conductance. The leakage channel is described by a voltageindependent conductance and the conductance of the other ion channels is voltage and time dependent.

If all channels are open, they transmit currents with a maximum conductance $g_{\mathrm{Na}}$ or $g_{\mathrm{K}}$, respectively. Normally, however, some of the channels are blocked. The probability that a channel is open is described by additional variables $m, n$, and $h$. The combined action of $\mathrm{m}$ and $\mathrm{h}$ controls the $\mathrm{Na}^{+}$ channels. The $\mathrm{K}^{+}$gates are controlled by $\mathrm{n}$. Hodgkin and Huxley formulated the three ionic components as

$$
\begin{aligned}
C \frac{d u}{d t}= & g_{\mathrm{Na}} m^{3} h\left(u-E_{\mathrm{Na}}\right)+g_{\mathrm{K}} n^{4}\left(u-E_{\mathrm{K}}\right) \\
& +g_{\mathrm{L}}\left(u-E_{\mathrm{L}}\right)+I(t) .
\end{aligned}
$$

The parameters $E_{\mathrm{Na}}, E_{\mathrm{K}}$, and $E_{\mathrm{L}}$ are the reversal potentials. Reversal potentials and conductances are empirical parameters. So, it is demonstrated that the neurons transmit information by firing and propagating electrical action potentials along their axons. Usually, motor neurons possess a myelin sheath around their axons acting as an insulator and enhancing the speed of signal transmission.

The model can reproduce and explain a wide range of data from squid axon like the shape and propagation of the action potential (see Fig. 3), its sharp threshold, refractory period, anode-break excitation, accommodation and sub threshold oscillations. The model can also describe many channel types with small parameter changes indicating the generality of the approach. Thus, the H-H model links the microscopic level of ion channels to the macroscopic level of currents and action potentials (see Fig. 4). 


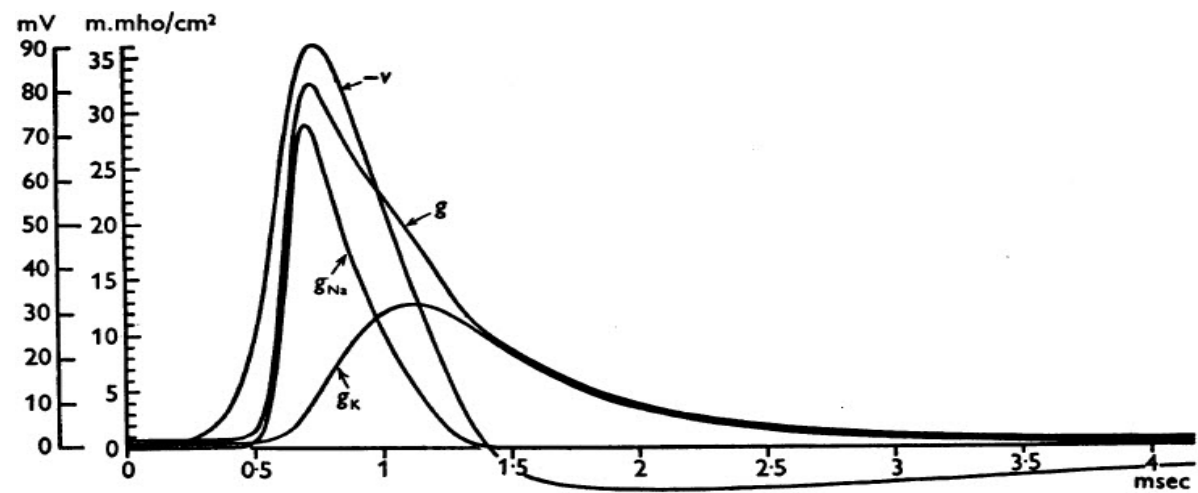

Fig. 4. Separation of ionic conductances underlying the action potential in H-H model. Modified from Hodgkin and Huxley (1952).

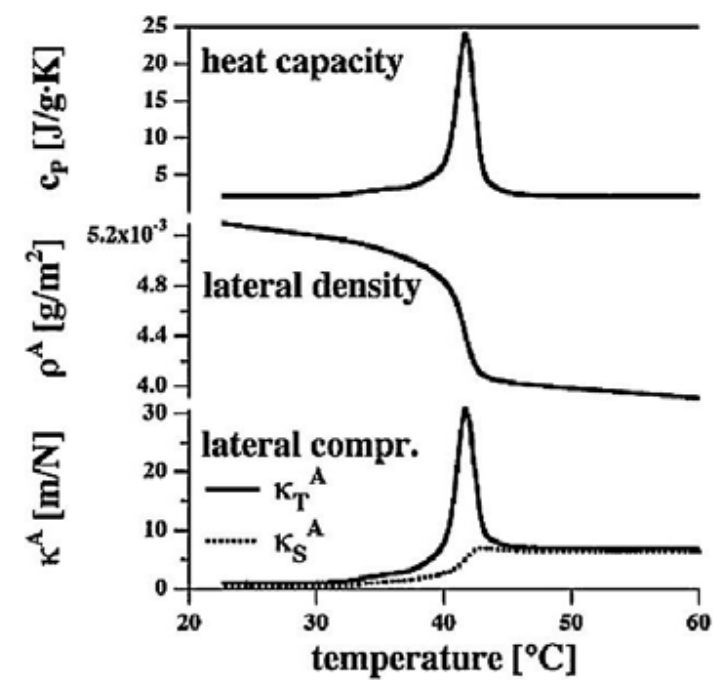

Fig. 5. Heat capacity, lateral density and lateral compressibility as a function of temperature (adapted from Heimburg and Jackson, 2005).

\section{Soliton theory}

T. Heimburg and A. D. Jackson proposed an alternative theory in 2005 . The theory is a thermodynamic theory of nerve pulse propagation. This theory is based on the lipid characteristic of transition from a fluid to a gel state over a range of several degrees, slightly below the body temperature (Heimburg and Jackson, 2005, and references there in). That is, when a biological membrane is compressed, the fluid membrane gradually reaches to a denser gel state. Now, a sufficient pressure/ compression either in membrane area or volume will bring a transition to the gel state (Heimburg, 2007a). This transition is associated with the release of heat (Heimburg, 2007a; Heimburg, 2007b). The relation between heat capacity, density, compression and temperature are shown in Fig. 5.
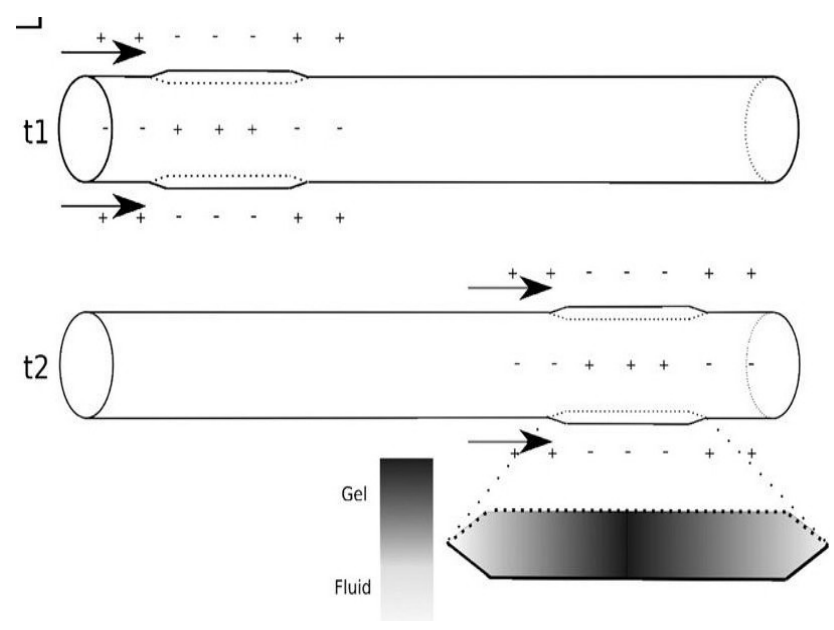

Fig. 6. Schematic model of membrane lipid thermodynamic theory (adapted from Anderson et al., 2009).

Lipid membranes have the properties required for the generation and propagation of solitons. Pure lipid membranes exhibit "non-linearity" (maximally compressible close to the transition and decreased compressibility with increasing density or pressure) and "dispersion" (lipid compressibility is frequency dependent both for area and volume compressibility) (Heimburg and Jackson, 2005; Heimburg and Jackson, 2007a).

Both the effects of non-linearity and dispersion produce a self-sustaining and localized density pulse with a moving segment of the nerve membrane in the gel state (see Fig. 6). This pulse is called a soliton (Heimburg and Jackson, 2007a). The solitary wave maintains its shape while it travels at a constant speed less than the sound velocity in the lipid membrane. Solitons can propagate over long distances without loss of energy (Heimburg and Jackson, 2005). The pulse is also called as adiabatic pulse, because no energy is lost to the environment during propagation (Heimburg and Jackson, 2007b). The soliton mode of pulse propagation is basically 
different from the AP given by the $\mathrm{H}-\mathrm{H}$ model (Anderson et al., 2009).

The solitary wave is associated with a transient voltage change due to the transient change in state of membrane. This affects the membrane potential difference. Especially, it changes both the area and thickness of membrane and thereby capacitance (Heimburg and Jackson, 2007a).

These changes seem to be as a voltage pulse and leads to a capacitive current due to the unbalanced charge of membrane. The reversible heat observed during action potential is proposed as a result of lipid melting enthalpy i.e. production of latent heat during lipid transition from fluid to gel state and the reabsorption of heat as the system returns to the fluid state (Anderson et al., 2009).

Thus the heat flow is correlated with the changes in membrane density and potential.

\subsection{Soliton equation}

Hydrodynamic equation for the propagation of a density pulse in the presence of dispersion for a cylindrical membrane along $\mathrm{x}$-axis is (Heimburg and Jackson, 2005) given by

$\frac{\partial^{2}}{\partial t^{2}} \Delta \rho^{A}=\frac{\partial}{\partial x}\left[c^{2} \partial / \partial x \Delta \rho^{A}\right]-h \frac{\partial^{4}}{\partial x^{4}} \Delta \rho^{A}$

After the co-ordinate transformation $(z=x-v \cdot t)$, by introducing the propagation velocity, the time independent equation describing the propagation density excitation is given as (Heimburg and Jackson, 2007a)

$$
\begin{aligned}
v^{2} \frac{\partial^{2}}{\partial z^{2}} \Delta \rho^{A}= & \frac{\partial}{\partial z}\left[\left(c 0^{2}+p \Delta \rho^{A}+q\left(\Delta \rho^{A}\right)^{2}+\ldots\right) \partial / \partial z \Delta \rho^{A}\right] \\
& -h \frac{\partial^{4}}{\partial z^{4}} \Delta \rho^{A}
\end{aligned}
$$

Where

$v$ is propagation velocity

$\rho^{A}$ is lateral membrane density

$c$ is sound velocity in fluid phase of membrane

$p$ and $q$ are the parameters determined from sound velocity and density dependence

$h$ is the parameter to set the linear scale of propagating pulse.

This theory also explains the effect of anesthetics on the nerve pulse propagation. According to this theory, the anesthetics in the membrane lower the fluid-gel transition temperature, a phenomenon known as freezing point depression (Heimburg and Jackson, 2007c). So it is difficult to force the membrane through the fluid-gel transition, thereby the action potential generation is suppressed. It is noted that freezing point depression is a general thermodynamic phenomenon and not chemical in nature (Anderson et al., 2009).

\section{Comparison}

The major differences between both the neural theories are (Hodgkin and Huxley, 1952; Heimburg and Jackson, 2005; Heimburg and Jackson, 2007a; Anderson et al., 2009):

$\mathrm{H}-\mathrm{H}$ The action potential is due to the electric current conducted across the membrane by ion channels that act as resistors.

$\mathrm{S}$ The nerve pulse is a solitary wave generated by the lipid transition in the membrane.

$\mathrm{H}-\mathrm{H}$ Nerve pulse propagation is then explained as a moving self regenerating pulse of local capacitive discharges through the resistors followed by capacitor recharging.

$\mathrm{S}$ The propagation is due to the combined effect of nonlinearity and dispersion.

$\mathrm{H}-\mathrm{H}$ The theory is pure electrical with ionic hypothesis.

$\mathrm{S}$ The theory is physical/ mechanical i.e. based on thermodynamics.

$\mathrm{H}-\mathrm{H}$ The nerve signal or action potential is electrical pulse.

$\mathrm{S}$ The nerve signal is propagating adiabatic electromechanical pulse.

$\mathrm{H}-\mathrm{H}$ The pulse propagation dissipates energy in the form of heat.

$\mathrm{S}$ The propagating pulse does not dissipate heat energy.

$\mathrm{H}-\mathrm{H}$ The nerve's physical changes are not explained.

$\mathrm{S}$ The nerve changes dimensions and gets thicker during nerve pulse. The voltage pulse accompanies the propagating signal, as a result of these transient geometric changes of the membrane.

Both the theories explain the existence of voltage pulse in nerve pulse propagation. However, as an information transmitting pulse in $\mathrm{H}-\mathrm{H}$ model and as a by-product signal in soliton theory.

\section{Discussion}

Hodgkin-Huxley model of nerve pulse propagation is undoubtedly a remarkable achievement in the field of physiology. However, the theory could not explain the physical phenomena such as reversible heat changes, density changes and geometrical changes observed in the experiments (Iwasa et al., 1980; Tasaki, 1982, 1999; Tasaki et al., 1989, Tasaki and Byrne, 1992).

Soliton model is an alternative model, convincingly explaining some of the unexplained observations in the experiments. However, there are several other questions that this 
has to answer like ion flow involvement in nerve signal propagation as stated by $\mathrm{H}-\mathrm{H}$ model and also the faster propagation in myelinated nerves than in unmyelinated.

\section{Context}

This is a theoretical comparison of both the theories. This has been carried out to analyze and choose a detailed neuron model which fits in for the goals of our project. We are examining the soliton theory to model and simulate the action potential and the coupling of electrode for a detailed study of the neuron.

Acknowledgements. We are grateful to DFG (German Science Foundation) for funding our project in the Research Training Group $1505 / 1$

\section{References}

Andersen, S., Jackson, A. D., and Heimburg, T.: Towards a thermodynamic theory of nerve pulse propagation, Progr. Neurobiol., 88, 104-113, 2009.

Bernstein, J.: Studies on the thermodynamics of bioelectric currents, Pflügers Arch. Ges. Physiol., 92, 521-562, 1902.

Cole, K. S. and Curtis, H. J.: Electric impedance of the squid giant axon during activity, J. Gen. Physiol., 22, 649-670, 1939.

Gerstner, W. and Kistler, M. W.: Spiking neuron models. Single neurons, populations, plasticity, Cambridge Univ. Press, p. 34, 2002.

Häusser, M.: The Hodgkin-Huxley theory of the action potential, Nature neuroscience, 3, 1165, doi:10.1038/81426, 2000.

Heimburg, T. and Jackson, A. D.: On soliton propagation in biomembranes and nerves, Proc. Natl. Acad. Sci. USA., 102, 9790-9795, 2005.
Heimburg, T.: Chapter 6: lipid melting, in: Thermal Biophysics of Membranes, Wiley-VCH, pp. 75-97, 2007a.

Heimburg, T. and Jackson, A. D.: On the action potential as a propagating density pulse and the role of anesthetics, Biophys. Rev. Lett., 2, 57-78, 2007a.

Heimburg, T.: Chapter 7: phase diagrams, in: Thermal Biophysics of Membranes, Wiley-VCH, pp. 99-122, 2007b.

Heimburg, T. and Jackson, A. D.: Thermodynamics of the nervous impulse. In: Nag, K. (Ed.), Structure and Function of Membranous Interfaces, Wiley and Sons, 2007b.

Heimburg, T. and Jackson, A. D.: The thermodynamics of general anesthesia Biophys. J., 92, 3159-3165, 2007c.

Hodgkin, A. L. and Huxley, A. F.: A quantitative description of membrane current and its application to conduction and excitation in nerve, J. Physiol., 117, 500-544, 1952.

Hodgkin, A. L.: The ionic basis of nervous conduction, Nobel Lectures, Physiology or Medicine, 1963-1970.

Hodgkin, A. L. and Katz, B.: The effect of sodium ions on the electrical activity of the giant axon of the squid, J. Physiol. (Lond.), 108, 37-77, 1949.

Iwasa, K., Tasaki, I., and Gibbons, R. C.: Swelling of nerve fibers associated with action potentials, Science, 210, 338-339, 1980.

Overton, E.: Contributions to general muscle and nerve physiology II. Indispensability of sodium (or lithium) ions for muscle contraction, Pflügers Arch. Ges. Physiol., 92, 346-386, 1902.

Tasaki, I.: Physiology and Electrochemistry of Nerve Fibers, Academic Press, New York, 1982.

Tasaki, I.: Evidence for phase transitions in nerve fibers, cells and synapses, Ferroelectrics, 220, 305-316, 1999.

Tasaki, I. and Byrne, P. M.: Heat production associated with a propagated impulse in bullfrog myelinated nerve fibers, Jpn. J. Physiol., 42, 805-813, 1992.

Tasaki, I., Kusano, K., and Byrne, P. M.: Rapid mechanical and thermal changes in the garfish olfactory nerve associated with a propagated impulse, Biophys. J., 55, 1033-1040, 1989. 